\title{
Cat Dander Antigen IgG Antibody Measurement
}

National Cancer Institute

\section{Source}

National Cancer Institute. Cat Dander Antigen Ig G Antibody Measurement. NCI

Thesaurus. Code C130125.

A measurement of the cat dander antigen IgG antibody in a biological specimen. 